\title{
Contrast Enhancement Of Satellite Images Based On Lifting Haar Wavelet Transform And Singular Value Decomposition
}

\author{
Dr.P.M.K.Prasad ${ }^{1}$, Dr.Y.Raghavender Rao $^{2}$, Dr.Karri Chiranjeevi ${ }^{3}$ \\ ${ }^{1}$ Associate Professor, Dept.of ECE, GVP College of Engineering for Women, Visakhapatnam, \\ Andhra Pradesh-530 048,India, pmkp70@gmail.com \\ ${ }^{2}$ Professor \& Head, Dept.of ECE, JNTUH College of Engineering, Sultanpur(V), Pulkal(M), Sangareddy(D), \\ Telangana-502 273, India, Email: yraghavenderrao@gmail.com \\ ${ }^{3}$ Post Doctoral Researcher, Dept of informatics, University of Beria Interior, Convento de Sto. António. 6201-001 \\ Covilhã. Portugal karri.chiranjeevi@ubi.pt
}

\begin{abstract}
For human visual system, contrast is one of the most important quality factors.. The existing contrast enhancement techniques such as contrast stretching, Histogram equalization can not only enhance the local contrast and global contrast of high contrast images, also enhances the edge and contour information of image, highlight the internal details of image and improve the visual effect. But, these methods are failed for low contrast images. The proposed method enhances the low contrast satellite images using Lifting Haar wavelet Transform and Singular Value Decomposition method. The input image decomposed in to four subbands with lifting discrete wavelet transform scheme. Then, the singular value matrix of the low-low subband image will be estimated and by applying inverse lifting wavelet transform to reconstruct enhanced image. The proposed method also compared with the existing traditional methods like contrast stretching, General Histogram Equalization (GHE), and Adaptive Histogram Equalization(AHE).The method is tested on different images. The results show that proposed method is superior to existing methods. So, for preventing the possible degradation in image and enhancing the illumination of image the lifting wavelet transform is applied. The reconstructed image is enhanced in terms of illumination. The various performance metrics like Mean Square Error(MSE), Peak Signal to Noise Ratio(PSNR), are compared for both the proposed method and existing methods. .
\end{abstract}

Key words : Contrast, Histogram Equalization, satellite image, wavelet transform

\section{INTRODUCTION}

Contrast enhancement plays an important role in many applications such as satellite image processing, medical image processing and astronomy etc. Satellite images are widely used in applications such as geosciences studies, and geographical information systems. Satellite images are usually low contrast images which has information, but it is not visible. Contrast is the most important quality factors in in human visual system. Contrast is the difference in luminance In visual perception, contrast is determined by the difference in the color and brightness of an object with other objects. The range of brightness values present on an image is referred to as contrast.. The satellite images are corrupted by noise during imaging procedures. Such as applications like enhancement of low contrast satellite images. The contrast enhancement techniques improves the visual quality of image by enhancing edge and contour information along with local, global contrast [1]. There are various contrast enhancement techniques such as contrast stretching[2], General Histogram Equalization (GHE)[3], Adaptive Histogram Equalization (AHE), Discrete Wavelet Transform. These contrast enhancement techniques are used for high contrast images only. But the proposed Lifting Haar Wavelet Transform with Singular Value Decomposition (SVD) is used for enhancement of low contrast images. The proposed method can preserve the probability distribution function of the image. In first step, discrete lifting Haar wavelet is applied which decomposes the low contrast satellite image in four sub bands LL, LH, HL, and HH. Then, the singular value matrix of the low-low sub band image is estimated and inverse lifting haar wavelet transform is applied to reconstruct enhanced image. The proposed method is compared with the existing methods and the results of the proposed method are superior when compared with the existing methods.

\section{CONTRAST ENHANCEMENT TECHNIQUES}

The most common problem in satellite imaging is that the range of reflectance values collected by a sensor may not match the capabilities of the film or color display monitor. The principal objective is to bring out details that are hidden in an image and to increase the contrast of an image. It sharpens image features such as edges, boundaries, or contrast to make a graphic display more helpful for display and analysis. Low contrast images can result from poor 
illumination, lack of dynamic range in the imaging sensor etc. If the contrast of an image is highly concentrated on a specific range, the information will be lost in those areas which are excessively concentrated. Various contrast enhancement algorithms were proposed to improve the quality of an image [18]. So, choosing an optimized contrast enhancement technique is necessary.

\subsection{Contrast Stretching}

Contrast Stretching is a linear function which increases the dynamic range of the gray levels in the image being processed. It is a process that expands the range of intensity levels in an image so that it spans the full intensity range of the display device[4]. This type of transformation is used to enhance low contrast images. In a low contrast image specific details are difficult to determine due to the fact that most pixels are at the same intensity value. In contract stretching, a low-contrast image exhibits small differences between its light and dark pixel values.

\subsection{General Histogram Equalization}

Histogram of an image represents the relative frequency of occurrence of gray levels to preserve mean brightness of the input image. The histogram in the context of image processing is the operation by which the occurrence of each intensity value in the image is shown. Histogram equalization is a scheme that improves the contrast of input image and stretch out the intensity range[5]. This method usually increases the global contrast of many images, especially when the usable data of the image is represented by close contrast values. Through this adjustment, the intensities can be better distributed on the histogram. The Histogram can be viewed graphically simply as a plot of ' $n$ ' versus 'rk' where rk is the kth gray level value and nk is the number of pixels with the kth gray level value. Histogram equalization (HE) is a well known and simple contrast enhancement technique[19][20]. General Histogram Equalization (GHE) works by flattening the histogram of input image and stretches dynamic range of gray levels by using cumulative density function (CDF) of the image. The GHE method re-maps the gray levels of input image by re-assigning intensity values of pixels to make a uniform intensity distribution. The method is useful in images with backgrounds and foregrounds that are both bright or both dark.

\subsection{Adaptive Histogram Equalization (AHE)}

Adaptive histogram equalization is a method of contrast enhancement which is different from ordinary histogram equalization. In adaptive method, many histograms are computed where each histogram corresponds to a different section of image. Hence, AHE improves the local contrast of an image and more details can be observed. With this method, information of all intensity ranges of the image can be viewed simultaneously. There are many ordinary display devices that are not able to depict the full dynamic intensity range. This method brings a solution to this problem. Other advantages are; it is automatic (i.e., no manual intervention is required) and reproducible from study to study. In AHE method, for each pixel in the input image, a region centred about a pixel is assigned. This region is called contextual region. The intensity values for this region are used to find the histogram equalization mapping function and this mapping function is applied to the pixel being processed in the region. The resultant image is produced where each pixel is mapped differently. Hence the intensities are distributed locally and contrast is enhanced based on the local area rather than the entire image. This method works too slowly on a general purpose computer although it works correctly. As enhancement is carried out in a local area, AHE tends to over enhance the noise content.

\subsection{Discrete Wavelet Transform}

When looking at a fourier transform of signal, it is impossible to tell when a particular event took place. So to overcome this drawback, the same transform was adapted to analyze only a small window of the signal at a time. This technique is known as short time fourier transform which maps a signal into a two dimensional function of time and frequency to get the localized point information but the only drawback is that, the window size is same for all frequencies. Many signals require more flexible approach that is flexible window size. This technique is known as wavelet transform.. It allows the different window sizes for different frequencies. Generally speaking, binary wavelet transform is often used in digital image processing. Wavelet transform is based on time-frequency analysis and analyze the image with different resolutions. DWT or DCT with SVD has been used for image contrast enhancement [12][13][14][15].

\subsubsection{Haar wavelet Transform}

In this paper, the Haar wavelet is selected, because it is basic and simple wavelet and it is more qualitative when compared with other wavelets. Haar wavelet function $\Psi(t)[6]$ is given by

$$
\Psi(t)= \begin{cases}1 & 0 \leq t \leq 1 / 2 \\ -1 & 1 / 2 \leq t \leq 1 \\ 0 & \text { elsew here }\end{cases}
$$

Haar scaling function defined as $\phi(t)$

$$
\phi(t)= \begin{cases}1 & 0 \leq t \leq 1 \\ 0 & \text { else }\end{cases}
$$

The wavelet expansion system is to be orthogonal across both translations and scale gives a clean, robust, and symmetric formulation with a Parseval's theorem [7]. When processing a 2-D image, the wavelet analysis is performed separately for the horizontal and the vertical directions. In the decomposition level 1 which is shown in the Figure 1, the image will be divided into 4 sub bands, called LH, LH, HL, and $\mathrm{HH}$ [8][9]. The LL sub band is a low-resolution residue that has low frequency components, which are often referred to as the average image. LH provides vertical detailed images. HL provides detailed images in the horizontal direction; The $\mathrm{HH}$ sub band image gives details on the diagonal. 
P.M.K.Prasad et al., International Journal of Emerging Trends in Engineering Research, 8(3), March 2020, 830- 837

\begin{tabular}{|l|l|}
\hline LL & LH \\
\hline HL & HH \\
\hline
\end{tabular}

Figure 1: First level Wavelet decomposition

\section{LIFTING HAAR WAVELET TRANSFORM}

The lifting Haar wavelet transform basic arithmetic is through a female wavelet to construct new wavelet gradually [10]. Consider a signal $s_{j}$ with $2^{\mathrm{j}}$ samples which we want to transform into a coarser signal $s_{\mathrm{j}-1}$ and a detail signal $\mathrm{d}_{\mathrm{j}-1 .}$. The lifting wavelet transform consists of three steps: split, predict and update.

\section{A) Split:}

The splitting is used for splitting the signal into two disjoint sets of samples. Here, one group consists of the even indexed samples $s_{21}$ and the other group consists of the odd indexed samples $\mathrm{s}_{21+1}$. Here, lifting process is implemented on basic Haar wavelet. Figure 2 shows the various steps in lifting Haar wavelet transform. An operator is built so that

$$
\left(\operatorname{even}_{\mathrm{j}-1} ; \operatorname{odd}_{\mathrm{j}-1}\right)=\operatorname{Split}\left(\mathrm{s}_{\mathrm{j}}\right)
$$

B) Predict:

The predict step uses a function that approximates the data set [11]. Even set is used to predict odd set. An odd sample $\mathrm{s}_{\mathrm{j}, 21+1}$ will use its left neighboring even sample $\mathrm{s}_{\mathrm{j}, 21}$ as its predictor. Let the detail $d_{j-1,1}$ be the difference between the odd sample and its prediction:

$$
\mathrm{d}_{\mathrm{j}-1,1, \mathrm{l}}=\mathrm{s}_{\mathrm{j}, 21+1}-\mathrm{s}_{\mathrm{j}, 21}
$$

which defines an operator $\mathrm{P}$ such that

\section{C) Update:}

$$
\mathrm{d}_{\mathrm{j}-1,1}=\operatorname{odd}_{\mathrm{j}-1}-\mathrm{P}\left(\mathrm{even}_{\mathrm{j}-1}\right)
$$

The even locations can be overwritten with the averages and the odd ones with the details. The operator $U$ of the form

$$
s_{j-1}=\text { even }_{j-1}+\mathrm{U}\left(d_{j-1}\right)
$$

Even Sequence

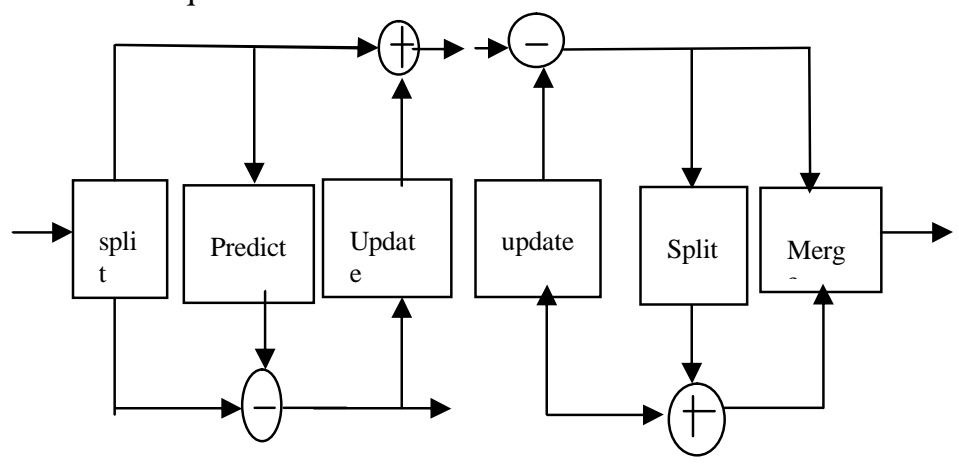

odd Sequence

Figure 2: Lifting Haar wavelet

\section{SINGULAR VALUE DECOMPOSITION}

Singular Value Decomposition(SVD) is a method for identifying and irdering the dimensions along which data points exhibit the most variation. Once it is identified where the most variation is present, then it is possible to find the best approximation of the original data points using fewer dimensions. Hence, SVD can be seen as a method for data reduction and mostly for feature extraction as well as for the enhancement of the low contrast images. In linear algebra, SVD is a factorization of a real or complex matrix. It is the generalization the eigen decomposition of a positive semi definite normal matrix (for example, a symmetric matrix with positive eigen values) to any matrix via an extension of polar decomposition. It has many useful applications in signal processing and statistics.Suppose $M$ is an $m \times n$ matrix whose entries come from the field $K$, which is either the field of real numbers or the field of complex numbers. Then there exists a factorization, called a singular value decomposition of $\mathrm{M}$, of the form

$$
\mathrm{M}=\mathrm{U \Sigma V}^{*}
$$

where $\mathrm{U}$ is an real or complex unitary matrix,$\Sigma$ is a $m * n$ rectangular diagonal matrix with non-negative real numbers on the diagonal and is $\mathrm{V}$ is an $\mathrm{n} * \mathrm{n}$ real or complex unitary matrix. The columns of $U$ and the columns of $\mathrm{V}$ are called the left-singular vectors and right-singular vectors of M respectively. The diagonal entries $\sigma_{i}$ of $\Sigma$ is known as the singular values of M. A singular value for which we can find two left (or right) singular vectors that are linearly independent is called degenerate. If $\mathrm{u} 1$ and $\mathrm{u} 2$ are two left-singular vectors which both correspond to the singular value $\sigma$, then any normalized linear combination of the two vectors is also a left-singular vector corresponding to the singular value $\sigma$. The similar statement is true for right-singular vectors. The number of independent left and right singular vectors coincides, and these singular vectors appear in the same columns of $\mathrm{U}$ and $\mathrm{V}$ corresponding to diagonal elements of $\Sigma$ all with the same value $\sigma$. In general, the SVD is unique up to arbitrary unitary transformations applied uniformly to the column vectors of both $\mathrm{U}$ and $\mathrm{V}$ spanning the subspaces of each singular value, and up to arbitrary unitary transformations on vectors of $\mathrm{U}$ and $\mathrm{V}$ spanning the kernel and co-kernel, respectively, of $\mathrm{M}$

\section{PROPOSED METHOD}

Figure 3 shows the block diagram of Lifting Haar Wavelet Transform(LHWT) using SVD method. $\mathrm{U}$ is a real or complex unitary matrix, $\Sigma$ is an $\mathrm{m} * \mathrm{n}$ rectangular diagonal matrix with non-negative real numbers on the diagonal and is $\mathrm{V}$ is an $\mathrm{n}^{*} \mathrm{n}$ real or complex unitary matrix. The columns of

$\mathrm{U}$ and the columns of $\mathrm{V}$ are called the left-singular vectors and right-singular vectors of $\mathrm{M}$ respectively. The diagonal entries $\sigma$ of $\Sigma$ is known as the singular values of M. So, for preventing the possible degradation in image and enhancing the illumination of image the Lifting Wavelet Transform is applied. After applying the inverse lifting wavelet one can get the reconstructed image. DWT -SVD based illumination enhancement presented by updating singular value matrix of singular value decomposition (SVD). Low-low(LL) sub-band of an input image is considered by applying DWT [16][17]. 


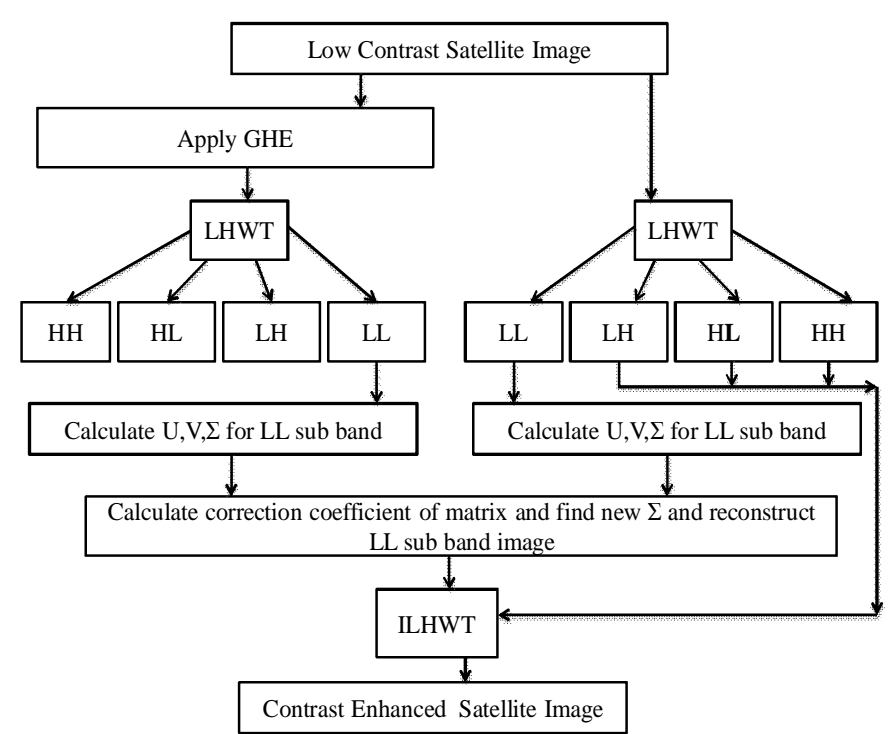

Figure 3: Block Diagram of Contrast enhancement of Satellite images using LHWT\& SVD method

The proposed LHWT and SVD based contrast enhancement method consists of following steps.

1. Apply General Histogram Equalization on the low contrast satellite image.

$\mathrm{A}=\mathrm{GHE}$ (low contrast satellite image)

2. Apply Lifting Haar Wavelet Transform to the GHE image and it decomposes GHE image into LL, LH, HL, $\mathrm{HH}$ bands which gives Approximation image, Horizontal, Vertical and Diagonal details of the image.

$$
\mathrm{B} \rightarrow \text { LHWT }(\mathrm{A}) \rightarrow[\mathrm{LL}, \mathrm{HL}, \mathrm{LH}, \mathrm{HH}]
$$

3. Apply Lifting Haar Wavelet Transform to the original image without applying GHE and generate the LL, LH, $\mathrm{HL}, \mathrm{HH}$ bands which give information regarding Approximation image, Horizontal, Vertical and Diagonal details of the image.

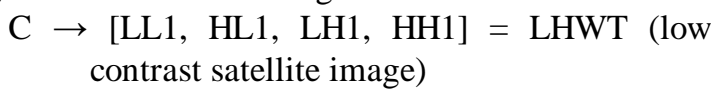

4. Apply Singular Value Decomposition matrix on LL band of LHWT of GHE image.

$$
\text { Matrix.1[S V D] = SVD (B.LL) }
$$

5. Apply Singular Value Decomposition matrix on LL band of LHWT of original image.

$$
\text { Matrix.2[S V D] = SVD }(\text { C.LL) }
$$

6. Calculate correction coefficient as the ratio of the maximum element in the diagonal matrix $S$ of Matrix 1 and the maximum element in the diagonal matrix $S$ of Matrix 2.

$$
\begin{aligned}
& \mathrm{R}=\max (\Sigma) \text { of Matrix.1[S V D] / } \max (\Sigma) \text { of } \\
& \text { Matrix.2[S V D] }
\end{aligned}
$$

7. Obtain the new Diagonal matrix as the product of error coefficient and the Diagonal matrix of Matrix 2

$$
\mathrm{D}=(\Sigma \text { of Matrix } 2) * \mathrm{R}
$$

8. Obtain the new approximate image as the product of new Diagonal matrix $\mathrm{D}$ and the column matrices $\mathrm{U}$ and $\mathrm{V}^{*}$ of the SVD matrix of original image.

$$
\mathrm{E}=\mathrm{UDV}^{*}
$$

9. Apply the inverse LHWT which contains new approximate image and the remaining detail information of original image.

\subsection{Performance metrics}

The PSNR block computes the peak signal-to-noise ratio, in decibels, between two images. The Mean Square Error (MSE) and the Peak Signal to Noise Ratio (PSNR) are the two error metrics used to compare image compression quality. The MSE represents the cumulative squared error between the compressed and the original image, whereas PSNR represents a measure of the peak error. The lower the value of MSE, the lower will be the error. To compute the PSNR, first calculates the mean-squared error using the following equation:

$$
P S N R=20 \log _{10} \frac{255}{\sqrt{M S E}}
$$

Where MSE is mean square error

$M S E=\frac{1}{m n} \sum_{x=1}^{m} \sum_{y=1}^{n}\left(I(x, y)-I^{\prime}(x, y)\right)^{2}$

$I(x, y)$ is original Image, $I^{\prime}(x, y)$ is contrast enhanced image. Here, $m$ and $n$ are the number of rows and columns in the input images, respectively.

\section{RESULTS AND DISCUSSION}

The low contrast satellite image whose contrast is to be enhanced is shown in Figure 4.
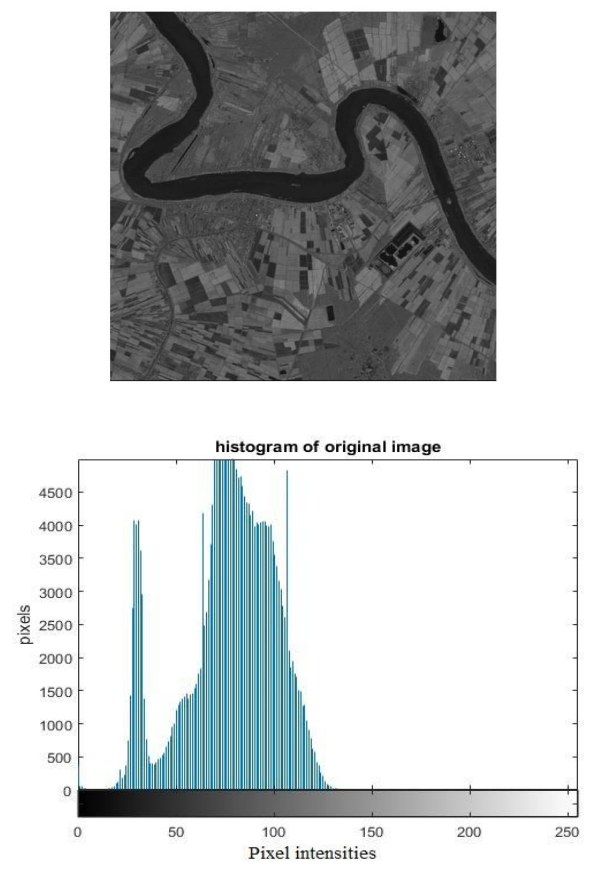

Figure 4 a. Low contrast satellite image b. histogram of low contrast satellite image 
The histogram of low contrast satellite image is inclined towards dark side of the histogram because of the uneven distribution of gray levels to the pixels. It can be seen that 125 pixels are given gray levels and some pixels are given maximum gray level intensities.
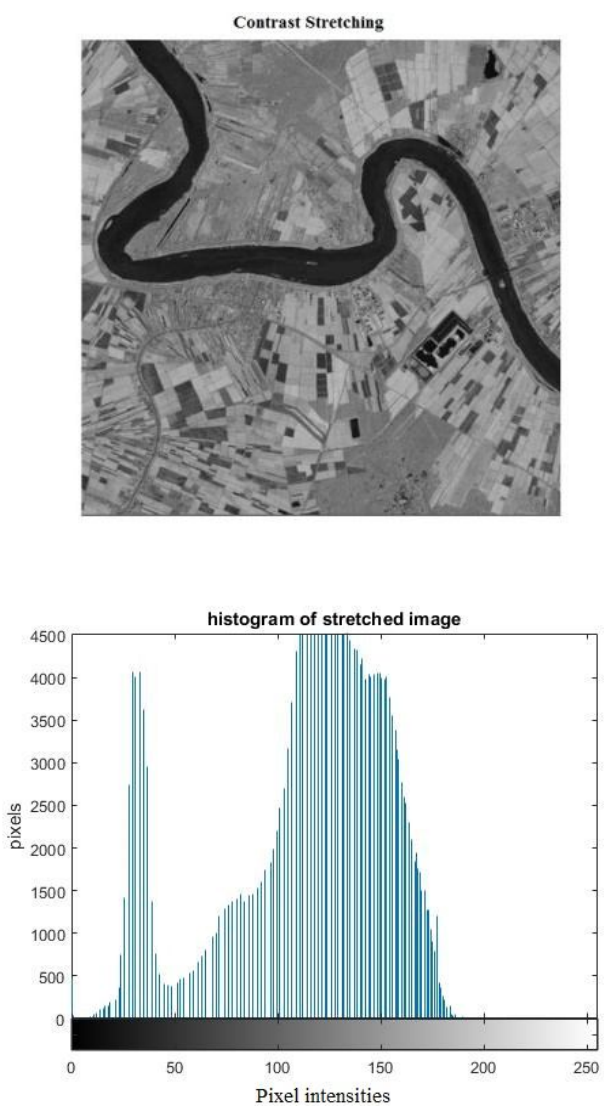

Figure 5.a. Contrast stretched image b. Histogram of contrast stretched image

The Contrast stretched image and it's resultant histogram is shown in Figure 5. It can be seen that histogram is increasing up to certain range and is decreasing and again increasing. It can be observed from the histogram that some pixels are given zero intensity and some pixels are given the maximum intensities. If the pixel intensities 0 and 255 are present in the image, enhancement never occurs.

Figure 6 represents the General Histogram Equalization of a low contrast satellite image. All the 255 pixel intensities are distributed. It can be clearly observed that some pixels are given zero gray levels which indicate the loss of background information as the enhancement is done globally.
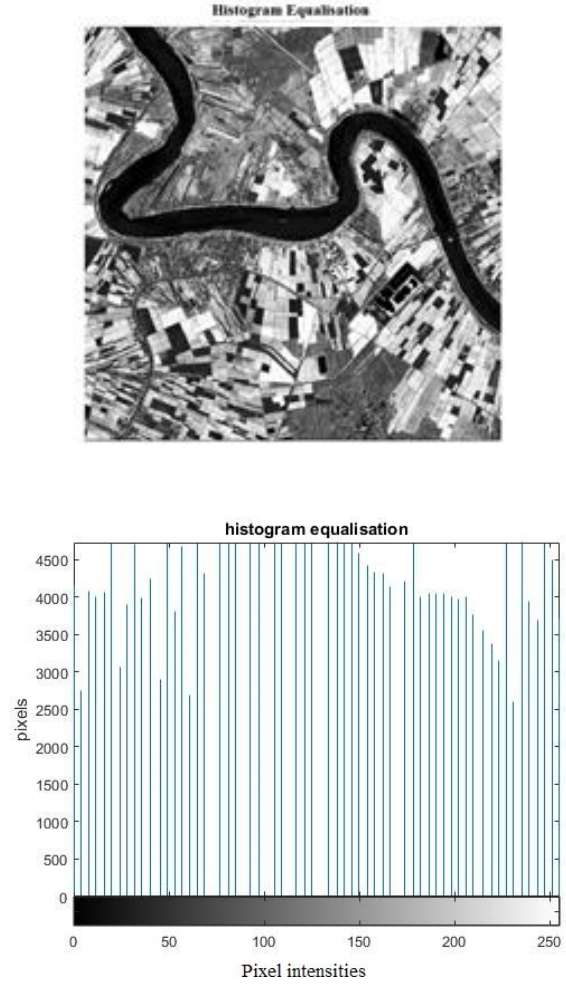

Figure 6 a. GHE image b. histogram of GHE image
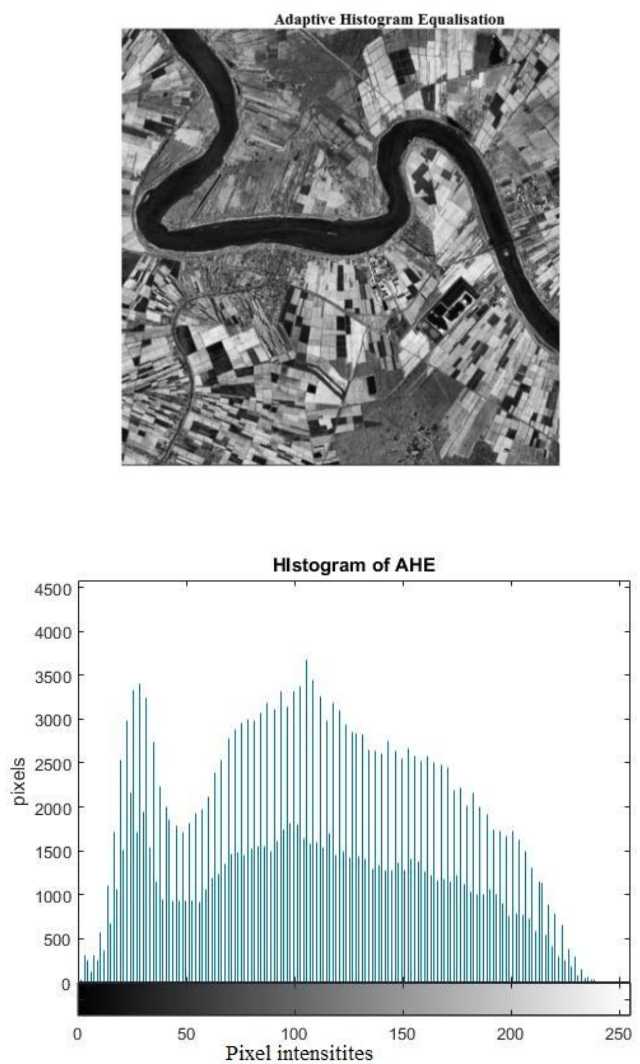

Figure 7 a. AHE of low contrast image b. Histogram of AHE image 
Figure 7 represents the Adaptive Histogram Equalization technique of a low contrast satellite image. It can be observed from the histogram that nearly 4000 pixels are distributed with intensities up to 240 . But the drawback is that noise is added because of over enhancement.

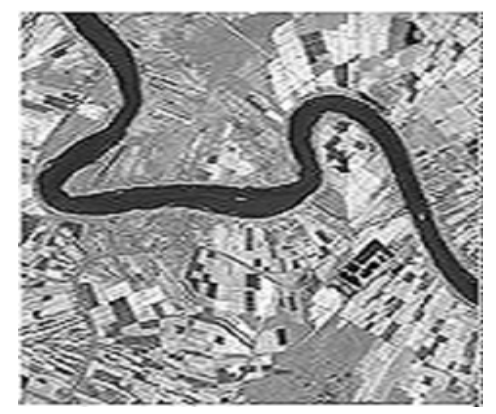

Figure 8. Contrast enhancement using DHWT

Figure 8 represents the sub bands of low contrast satellite image obtained by applying Discrete Haar Wavelet Transform. After applying the wavelet transform the whole image is decomposed into four different sub bands and each sub band has different significance. The Low-Low sub band contains average information of the image and other three sub bands ( $\mathrm{LH}, \mathrm{HL}$, and $\mathrm{HH}$ ) contain the detail coefficients of image. These three sub bands give the information about edges. The LL sub band gives the illumination information of image. So, for preventing the possible degradation in image and enhancing the illumination of image the Lifting Haar Wavelet Transform is applied. After applying the inverse lifting wavelet one can get the reconstructed image.
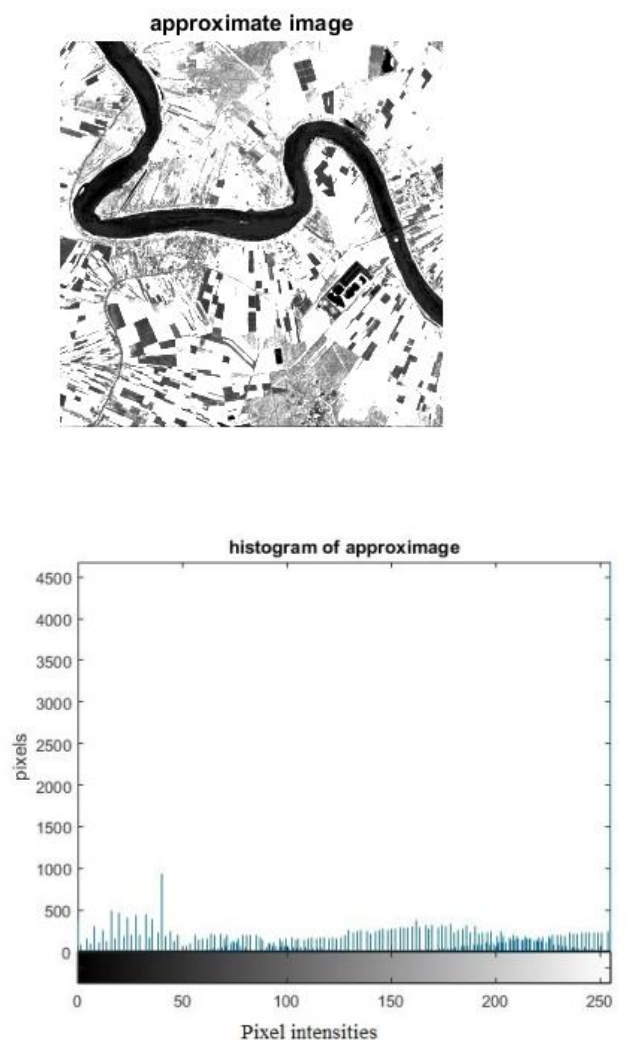

Figure 9.a. Approximate image of GHE b. histogram

Figure 9 represents the approximation image obtained by applying Lifting Wavelet Transform on the resultant GHE image. It contains the maximum information of the image and all the other sub bands contain the details of the image. It can be seen that all pixel intensities are distributed and nearly

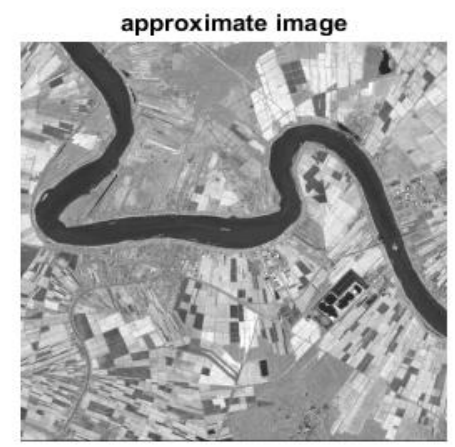

4500 pixels have the maximum intensity.

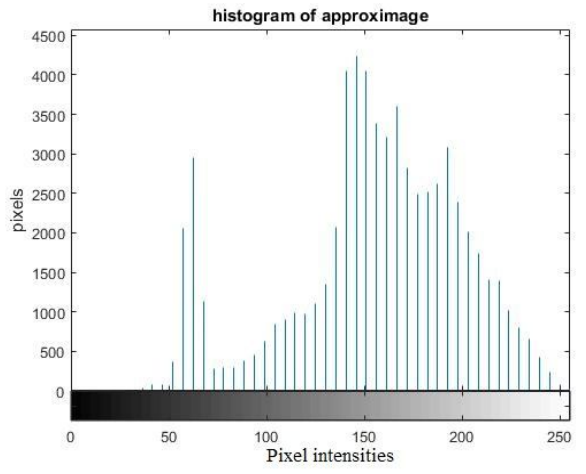

Figure 10 a. Approximate image obtained by applying LWT on low contrast image b. Histogram

Figure 10 represents the approximation image obtained by applying Lifting Wavelet Transform on low contrast satellite image. It can be seen that nearly 4000 pixels have intensity of 150 and the histogram is shifted right which increases the contrast.

Figure 11represents the resultant image obtained by applying SVD on the LL band of original image. It can be seen that histogram has shifted towards right compared to the original image. This indicates the enhancement of contrast. 

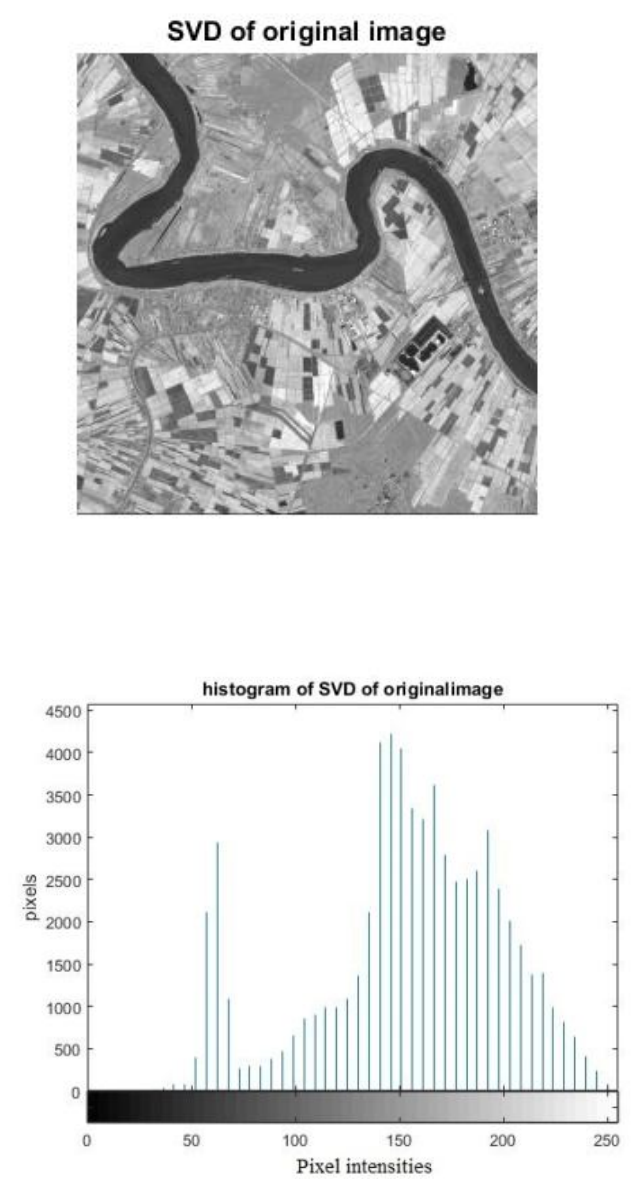

Figure 11 a. SVD of LL band of low contrast image b. its histogram

Figure 12 represents the enhanced image which is obtained by applying the combination of Lifting Wavelet Transform and SVD methods. It can be clearly observed that compared to stretching, GHE, AHE, this method gives better result without the addition of noise and even background information is not lost. Histogram has shifted right compared to the original image. This results in the contrast enhancement.

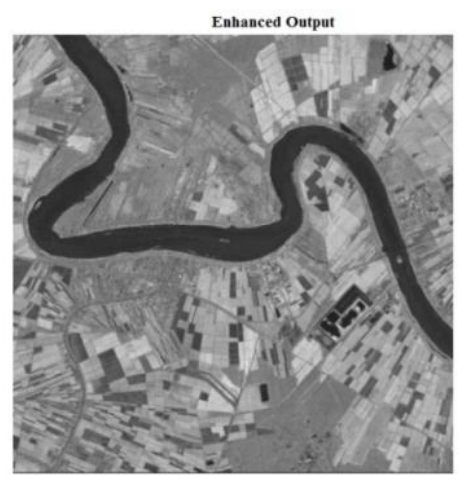

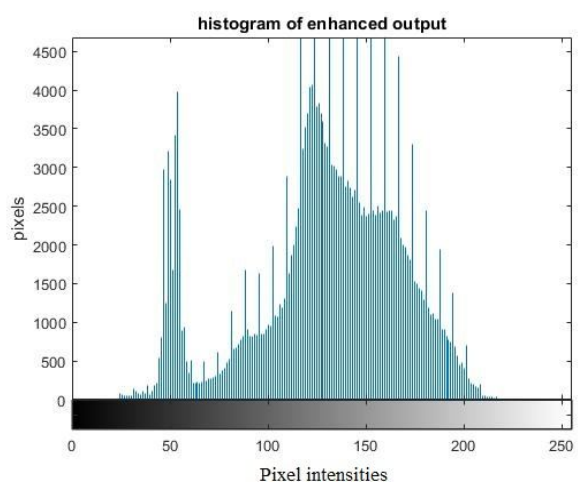

Figure 12 a. Enhanced output by applying LHWT using SVD method b. its histogram

Table 1: Comparison of MSE and PSNR

\begin{tabular}{|c|c|c|}
\hline Method & MSE & PSNR (dB) \\
\hline Contrast Stretching & 16.4890 & 42.0788 \\
\hline GHE & 14.4573 & 42.9853 \\
\hline AHE & 7.5309 & 45.7806 \\
\hline DHWT & 16.3859 & 42.0924 \\
\hline $\begin{array}{c}\text { Proposed LHWT with } \\
\text { SVD }\end{array}$ & 5.4614 & 46.7783 \\
\hline \multicolumn{2}{|c|}{} \\
\hline
\end{tabular}

Table 1 shows comparison of MSE and PSNR of proposed method with existing methods. It can be seen from the table. 1 that Contrast Stretching has more Mean Square Error which results in the degradation of PSNR. The proposed technique LHWT using SVD is more superior method since it has low Mean Square Error and high PSNR compared to existing methods.

\section{CONCLUSION}

In this paper, the proposed Lifting Haar Wavelet transform with Singular value Decomposition is applied for contrast enhancement of satellite images. The proposed Lifting Wavelet Transform and SVD based method increases the contrast without any loss of information. When SVD is applied on image, the resultant singular value matrix contains the illumination information of the image. So, if the singular values of matrix are changed, then the illumination of image also directly affected but other information of image will remain unchanged. So, for preventing the possible degradation in image and enhancing the illumination of image the lifting wavelet transform is applied. The proposed method was compared with the existing methods such as GHE, AHE, Contrast Stretching, DWT. The results shows that the proposed method is an efficient method for enhancement of low contrast satellite images both in terms of visual quality as well as performance metrics such as PSNR and MSE. 
P.M.K.Prasad et al., International Journal of Emerging Trends in Engineering Research, 8(3), March 2020, 830- 837

\section{REFERENCES}

1. Aswathy Mohan, Mary Linda PA, Image Enhancement Using DWT DCT and SVD, International Journal of Engineering Research and Applications ISSN : 2248-9622, Volume 4, Issue 4, pp 36-40 April 2014.

2. Koushlendra K Singh, Rajesh K. Pandey, Suraj Suman, Contrast Enhancement Using Lifting Wavelet Transform, IEEE International Conference on Control, Instrumentation, Communication and Computational Technologies,10-11July 2014. Kanyakumari,India, DOI: 10.1109/ICCICCT.2014.6993003

3. Neena K.A, Aiswarya Raj, Rajesh Cherian Roy, Image Enhancement based on Stationary Wavelet Transform, Integer Wavelet Transform and Singular Value Decomposition, International Journal of Computer Applications, Volume 58, Number 11, 2012, DOI:10.5120/9327-3632

4. R.C. Gonzalez and R. E. Woods, Digital Image Processing. Englewood Cliffs, NJ: Prentice-Hall, 2007

5. Ayesha Nasir, Nighat Shaheen, Kamran Ameen, Muhammad Adnan Khan, 5Muhammad Nadeem Ali Contrast Enhancement Using Histogram Equalization, Proceedings of the 3rd International Conference on Engineering \& Emerging Technologies (ICEET), Superior University, Lahore, PK, 7-8 April, 2016.

6. K.P.Soman,K.I.Ramachandran, Insight into wavelets from theory to practice, second edition Prentice Hall of India,2008.

7. P.M.K.Prasad, M.N.V.S.S.Kumar, G.Sasi Bhushana Rao Design of Biorthogonal wavelets based on parameterized filter for the analysis of X-ray images Computational Intelligence in Data Mining, Volume 2, Smart Innovation, Systems and Technologies 32,pp 99-110, Springer India 2015, DOI 10.1007/978-81-322-2208-8_11.

8. M. Antonini, M. Barland, P. Mathien and I. Daubechies, Image coding using wavelet transform, IEEE Trans. Image Processing, vol. 1, pp.205-220,April 1992.

9. S.Jayaraman Esakkirajan, T. veerakumar "Digital Image Processing Tata Mc Graw Hill, publication 2009.

10. S Devendra, P.M.K.prasad, Lifting Biorthogonal Wavelet Design for Edge Detection, IEEE International conference on High Performance Computing and Applications (ICHPCA) DOI: 10.1109/ICHPCA.2014.7045368, CV Raman college of Engineering, Bhubaneswar, odisha, India, 22-24 December 2014.

11. T. Prabhakara Rao, Dr. B. Rama Rao, Image Fusion using Lifting Wavelet Transform with Neural Networks for Tumor Detection, International Research Journal of Engineering And Technology, Volume: 06 Issue: 09| Sep 2019.
12. H. Demirel, C. Ozcinar, and G. Anbarjafari, Satellite image contrast enhancement using discrete wavelet transform and singular value Decomposition, IEEE Geoscience and remote sensing letters, vol. 7, no. 2, pp. 333-337, 2010.

13. R. Atta, and R.F. Abdel-Kader. Brightness preserving based on singular value decomposition for image contrast enhancement, Elsevier, Optik 126, pp. 799 803, 2015.

14. R. Atta, and M. Ghanbari. Low-contrast satellite images enhancement using discrete cosine transform pyramid and singular value decomposition, IET Image Processing, vol. 7, issue 5, pp. 472-483, 2013.

15. A.K. Bhandari, A. Kumar, and P.K. Padhy. Enhancement of low contrast satellite images using discrete cosine transform and singular value decomposition, International Journal of Computer, Electrical, Automation, Control and Information Engineering, vol. 5, no. 7, pp. 707-713, 2011.

16. Bhandari A K, Gadde M, Kumar A, Singh, GK. Comparative analysis of different wavelet filters for low contrast and brightness enhancement of multispectral remote sensing images, Proceedings of the IEEE international conference on machine vision and image processing (MVIP), 2012.

17. Bhandari , A.K.; Kumar, A.; Padhy, P.K Enhancement of low contrast satellite images using discrete cosine transform and singular value decomposition World AcadSciEng Technology 2011; 79:35-41.

18. Z. Y. Chen, B. R. Abidi, D. L. Page, and M. A. Abidi, Gray-level grouping (GLG): An automatic method for optimized image contrast enhancement-Part I: The basic method, IEEE Trans. Image Process., vol. 15, no. 8, pp. 2290-2302, Aug. 2006.

19. S. A. Stark, Adaptive image contrast enhancement using generalizations of histogram equalization, IEEE Trans. Consum. Electron., vol. 9, no. 5, pp. 889-896, May 2000.

20. J. Y. Kim, L. S. Kim, and S. -H. Hwang, An advanced contrast enhancement using partially overlapped sub-block histogram equalization, IEEE Trans. Circuits Syst. Video Technol., vol. 11, no. 4, pp. 475-484, Apr. 2001 